\title{
Doppler Shift Measurements Using Monte Carlo Modeling
}

\author{
Ren-Chiann Chian, Bor-Shyh Lin, Bor-Shing Lin, Fok-Ching Chong \\ Institute of Electrical Engineering, National Taiwan University, Taiwan, R.O.C.
}

\begin{abstract}
:
The most desired aim of clinical detection is to minimize the dimension of the detection instruments but still maintain high resolution and high precision. One of the most important mechanisms for maintaining the life of human beings is the human circulatory system. Thus, this researches focus on a non-invasive technique of photon measuring. From the measured result we could obtain important biomedical parameters for pathological diagnosis.

This project is to implement a non-invasive optical flow velocimetry for detecting the human circulatory system under the skin surface. Then numerical simulation and model analysis of the transmission of photon on the skin surface is calculated via Monte Carlo Method. The source of the incidence photon is He-Ne laser. The signal is transmitted and detected via Y-type optical fiber. Optical heterodyning is used to measure the frequency difference between the reflection wave and the original incidence laser wave. This is a real-time and continuous detecting blood flow velocimetry. We find that this is a reliable tool for doctors when doing clinical diagnosis.
\end{abstract}

Key words: clinical detection, circulatory system, Monte Carlo Method, He-Ne laser, optical heterodyning.

\section{Introduction:}

In recent year, the use of optics in biomedical engineering field is developing rapidly. Optical electrical detection techniques [1,2] with high resolution and high precision have become the important aim of many research institutes, especially in minimizing the dimension of clinical detection devices and in making it non-invasive. To attain this goal, the use of optical fiber has become one of the most important and unexpendable methods. For example, we can use non-invasive optical oximetry [3] and fiber-optic fluorometer [4] to analyze the human organizations. We can use hematoporphrin to detect tumor [5], and optical fiber is used to analyze the various components in blood [6], such as $\mathrm{pH}$ value, $\mathrm{PO}_{2}, \mathrm{PcO}_{2}$ etc. Even that in the newest research project, the use of non-invasive optical detection of blood sugar [7] is being paid attention by many scholars in this field.

Blood flow velocity is one of the important parameters of human circulatory system [8]. In recent year there have been many methods proposed by many specialists to detect this parameter via skin surface. However, most of these methods use indirect way to detect this parameter and cannot provide continuous detection. This will result in some restriction for their usage in some special applications. In recent research, coherent monochromatic laser light is used to incident the skin surface directly. This provides an application in clinic research on blood flow velocity via the Doppler effect due to fluid particles being lightened by the incidence laser. This method can measure the velocity of blood directly and it can serve as a continuous non-invasive detection technique with good resolution.

The earliest application of Doppler effect to detect blood flow velocity is published by Riva [9] 
and his group in the study of blood flow of Rabbits' retina capillary in 1972. In 1974, Tanaka's group [10] extends it to the experiment of human body. One year later they use optical fiber to detect blood flow velocity. In the same year, Sterm [12] published his research on the topic of micro circulatory system detection. In 1978, the first detection system of blood flow velocity on skin surface using laser Doppler effect is implemented by Watkin and Holloway [13]. There are many papers about how to improve the performance of the detection system and about the associate applications on clinical detection and diagnosis. For example, laser Doppler velocimetry is used for clinical diagnosis in retina capillary, and laser Doppler image is used to detect micro circulatory system on skin surface [14-17].

In the research field of circulatory system detection, biophotonics technique has become more and more important. For this reason, we hope to improve the applications of optical electronics in medical field. This project is to implement a Laser Doppler blood flow velocimetry for detecting the micro circulatory system under skin surface. Then numerical simulation and analysis by Monte Carlo method [18] is used to evaluate the performance of the Laser Doppler velocimetry in human body. This research is also conducted in clinical animal experiment. This result also reveals the associate mechanism about circulatory system of heart and body surface. We intend to promote the use of this velocimetry as an efficient detection tool to doctors in National Taiwan University Hospital.

The blood flow velocimetry is design and is implemented with a configuration consists of fiber, laser fiber driver, and signal amplifier laser Doppler effect is used as a guideline. Besides this, we do mathematical simulation and signal analysis and processing. Besides constructing a real-time and continuous detection tool, our main research aim is to find a more effective and reliable tool to help doctors to do clinical diagnosis.

\section{Theory and Method:}

When we use coherent laser light to focus on some moving object, the light wave of reflection will change its frequency when the relative location and the velocity of this moving object change. This relation is as follows:

$f=(1 / 2 \pi)\left(\mathrm{K}_{\mathrm{s}}-\mathrm{K}_{\mathrm{i}}\right) \cdot \mathrm{V}$

$\mathrm{K}_{\mathrm{s}}$ and $\mathrm{K}_{\mathrm{i}}$ are the propagation vectors of scatter beam and incident laser beam respectively, $\mathrm{V}$ is the velocity of the moving object and $f$ is the frequency change of the reflective wave. Fig 1.shows the optical setup for the two-fiber laser Doppler anemoment. The polarized $\mathrm{He}-\mathrm{Ne}$ laser beam is divided into incident and reference beams by the $50 / 50$ polarized beam splitter (BS). The reference light is coupled into Acousto-Optic Modulator, shifting of the reference frequency by $4 \mathrm{MHz}$ $(f+4 \mathrm{MHz})$.The incident light is coupled into the transmission fiber via the fiber-optic coupler. Based on Doppler effect, the scatter light frequency shifts by $\mathrm{d} f(f+\mathrm{d} f)$ when the incident light goes though the transmission to the catheter and through the skin surface into the red blood cell ( RBC). Therefore we apply the optical heterodyning method to get the blood flow velocimetry.

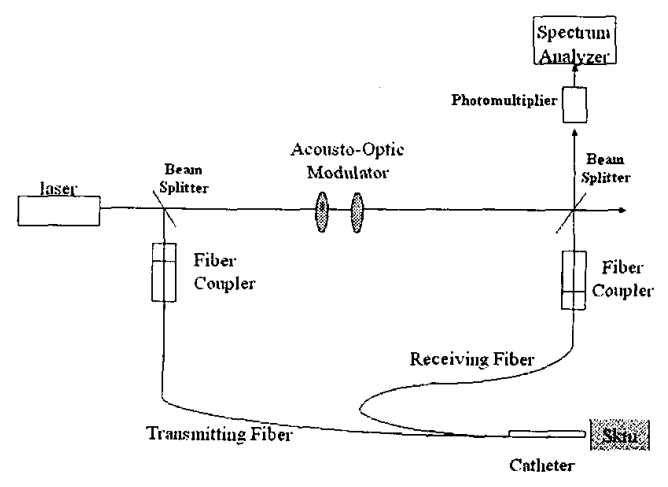

Fig. 1 Schematic diagram of the two-fiber Laser Doppler Anemoment 
Fig. 2 is the sectioned view of the fiber catheter. In this figure, the transmission fiber is perpendicular to the arm and the receive fiber is at an angle $\theta$ with the arm. Please note that the catheter does not directly contact to the arm. Consequently, there are three parameters in this optical system namely $\triangle f, \theta$ and the distance form catheter to the arm. Monte Carlo method is used to obtain the optimum state. The whole system is then calibrated.

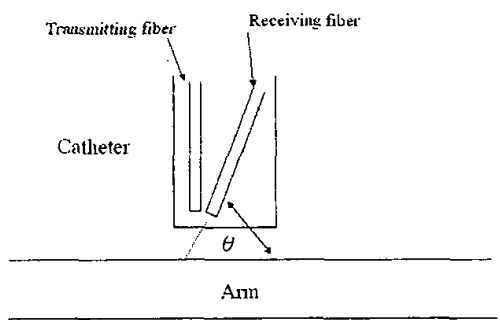

Fig. 2 Sectioned view of the fiber catheter

\section{Result:}

The configuration of fig. 1 is first calibrated. Paper is used in the calibration before it was experimented with human being. The paper is vibrated as a dangling object. The result displaying on the oscilloscope is recorded and shown in table 1. Likewise the above experiment is repeated on the tail of the rat.

Table 1

\begin{tabular}{c|c|c|c}
\hline & \multicolumn{3}{c}{ Oscilloscope $(\mathrm{mV})$} \\
\hline & 1st reading & 2nd reading & 3th reading \\
\hline The paper at still & 5 & 4.8 & 4.3 \\
\hline $\begin{array}{c}\text { The paper in } \\
\text { vibration }\end{array}$ & 45 & 43 & 41 \\
\hline Tail of Rat & 23 & 29 & 24 \\
\hline
\end{tabular}

Theoretically, using Doppler effect, we can calculate the velocity of the moving object. Then we apply the Monte Carlo method to modulate this system to the optimum state and obtain the velocity of the blood stream.

\section{Conclusion and discussion:}

From the analysis of the experimental data, we conclude that the system is capable of measuring the velocity of the red blood cell. There are many parameters involved that will interfere correct reading using laser Doppler effect. However, we find that through the use of Monte Carlo simulation, we can simulate the system in parallel and obtain good results. This method is efficient, cost saving and reliable.

\section{References:}

1. J.G. Webster, et. al., "Encyclopedia of medical devices and instrumentation: Fiber optics in medicine", New York: John Wiley \& Sons, vol. 2. pp. 1284-1302, 1988.

2. J.G.. Webster, et. al., "Encyclopedia of medical devices and instrumentation: Optical sensors" New York: John Wiley \& Sons, vol.3, pp.2121-2134, 1988 .

3. E. H. Wood and J. E. Geraci, "Photoelectric determination of arterial oxygen saturation in man. "J. Lab. Clin. Med., vol.34, pp387, 1949 •

4. A. Mayevsky and B. Charnce, "Intracellular oxidation-reduction state measured in situ by multichannel fiber-optic surface fluorometer." Science, vol. 217, pp.537, 1982.

5. D. R. Doiron and G. S, Keller, "Porphyrin photodynamic therapy: Principles and clinical applications." Curr. Probi. Dermatol., vol. 15, pp. 85, 1986. 
6. D. W. Lubbers, "Blood gas analysis with fluorescent dyes as an example of their usefulness as quantitative chemical sensors." Anal. Chem. Symp. Ser., vol. 17, pp.609-619, 1983."

7. F. M. Ham, 1. V. Kostanic, G. M. Cohen, and B. R. Gooch. "Determination of glucose concentrations in an aqueous matrix from NIR spectra using optimal time-domain filtering and partial least-squares regression." IEEE Trans. Biomed. Eng. Vol. 44, no. 6, 1997.

8. J. G. Webster, "Medical instrumentation: Application and design." 2'nd edition, Houghton Mimin, 1992.

9. C. Riva, B.Boss, and G. B. Benedek, "Laser Doppler measurements of blood flow in capillary tubes and retinal arteries." Invest. Ophthalmol, vol. II, pp. 936; 1972.

10. T. Tanaka. C. Riva, and 1. Ben-Sira, "Blood velocity measurements in human retinal vessels." Science, vol. 186, PP.830. 1974.

11. 11. T. Tanaka and G. B. Benedek, "Measurement of the velocity of blood flow (in vivo) using a fiber-optic catheter and optical mixing spectroscopy." Appl. Opt, vol.14, pp.189, 1975.

12. 12. M. Stern, "In vivo evaluation of mocrocirculation by coherent light scattering." Nature (London), vol. 254, pp.56, 1975.

13. D. W. Watkins and G. A. Holloway, "An instrument to measure cutaneous blood flow using the Doppler shift of laser light." IEEE Trans. Biomed. Eng., vol.25, pp.28. 1978.

14. J.E. Grunwald, C.E. Riva, S.H. Sinclair, A.J. Brucker, and B.L. Petrig," Laser Doppler velocimetry study of retinal circulation in diabetes mellitus." Archives of Ophthalmology, vol.104, no.7, pp.991-996, 1986.

15. N. Fujio, A. Yoshida, H. Ogasawara, G.T. Feke, and J.W. McMeel," The new laser Doppler velocimetry for the measurement of retinal circulation and its clinical application." Hokkaido Journal of Medical Science, vol. 71, no. 6, pp.757-769, 1996.

16. C. Svedman, G.W. Cherry, E. Strigini, T.J. Ryan, " Laser Doppler imaging of skin microcirculation." Acta Dermato-Venereologica. vol. 78, no.2, pp, 114-118, 1998.

17. C. Picart, P,H. Carpentier, S. Brasseur, H. Gailiard, J.M. Piau, "Systemic sclerosis: blood rheometry and laser Doppler imaging of digital cutaneous microcirculation during local cold exposure," Clinical Hemorheology \& Microcirculation. Vol. 18, no. 1, pp.47-58, 1998.

18.Maurice F. Aburdene, "Computer Simulation of Dynamic Systems", Chap. 8,1998

\section{Acknowledgements}

The authors would like to thank National Science Council for our experiments. This work is supported by NSC 89-2213-E-002057. 